Research in Astron. Astrophys. Vol.0 (200x) No.0, 000-000

http://www.raa-journal.org http://www.iop.org/journals/raa

Research in

Astronomy and

Astrophysics

Key words: galaxies: interactions — galaxies: groups: general

\title{
A sample of galaxy pairs identified from the LAMOST spectral survey and the Sloan Digital Sky Survey
}

Shiyin Shen ${ }^{1,2}$, M. Argudo-Fernández ${ }^{1}$, Li Chen ${ }^{1}$, Xiaoyan Chen $^{3}$, Shuai Feng ${ }^{1,4}$, Jinliang Hou ${ }^{1}$, Yonghui Hou ${ }^{5}$, Peng Jiang ${ }^{6}$, Yipeng Jing ${ }^{7}$, Xu Kong ${ }^{8}$, Ali Luo ${ }^{3}$, Zhijian Luo ${ }^{2}$, Zhengyi Shao ${ }^{1,3}$, Tinggui Wang ${ }^{6}$, Wenting Wang ${ }^{8}$, Yuefei Wang ${ }^{5}$, Hong $\mathrm{Wu}^{3}$, Xue-Bing $\mathrm{Wu}^{9}, 10$, Haifeng Yang ${ }^{3}$, Ming Yang ${ }^{3}$, Fangting Yuan ${ }^{1,2}$, Hailong Yuan $^{3}$, Haotong Zhang ${ }^{3}$, Jiannan Zhang ${ }^{3}$, Yong Zhang ${ }^{5}$ and Jing Zhong ${ }^{1}$

${ }^{1}$ Key Laboratory for Research in Galaxies and Cosmology, Shanghai Astronomical Observatory, Chinese Academy of Sciences, 80 Nandan Road, Shanghai, 200030, China, ssy@shao.ac.cn;

2 Key Lab for Astrophysics, Shanghai, 200234, China;

3 National Astronomical Observatories, Chinese Academy of Sciences, Beijing, 100012, China;

${ }^{4}$ University of Chinese Academy of Sciences, Beijing, 100049, China;

5 Nanjing Institute of Astronomical Optics \& Technology, National Astronomical Observatories, Chinese Academy of Sciences, Nanjing 210042, China

6 University of Science and Technology of China, Hefei, 230026, China;

7 Department of Physics and Astronomy, Shanghai Jiao Tong University, Shanghai, 200240, China;

8 Institute for Computational Cosmology, University of Durham, South Road, Durham, DH1 3LE, UK

9 Department of Astronomy, Peking University, Beijing, 100871, China;

10 Kavli Institute for Astronomy and Astrophysics, Peking University, Beijing 100871, China

\begin{abstract}
A small fraction( $<10 \%)$ of SDSS main sample galaxies(MGs) have not been targeted with spectroscopy due to the the fiber collision effect. These galaxies have been compiled into the input catalog of the LAMOST extra-galactic survey and named as the complementary galaxy sample. In this paper, we introduce the project and the status of the spectroscopies of the complementary galaxies in the first two years of the LAMOST spectral survey(till Sep. of 2014). Moreover, we present a sample of 1,102 galaxy pairs identified from the LAMOST complementary galaxies and SDSS MGs, which are defined as that the two members have a projected distance smaller than $100 h_{70}^{-1} \mathrm{kpc}$ and the recessional velocity difference smaller than $500 \mathrm{~km} \mathrm{~s}^{-1}$. Compared with the SDSS only selected galaxy pairs, the LAMOST-SDSS pairs take the advantages of not being biased toward large separations and therefor play as a useful supplement to the statistical studies of galaxy interaction and galaxy merging.
\end{abstract}




\section{INTRODUCTION}

In the standard hierarchical structure formation model, galaxies are built up through merging processes. Numerical simulations show that the galaxy mergers can trigger the star burst, feed the central super-massive black hole and transform the galaxy morphology (Springel et al., 2005). These different physical processes take places at different stages of galaxy merging. At early stage, as two galaxies approaching, they start to have interactions on each other out to a distance of about $100 \mathrm{kpc}$. After the first passage, the galaxies start to show strong tidal tails and undergo star bursts. After few times of passages, the galaxies quickly evolve into final coalescence. The whole time scale of the merging progress takes bout $1-2$ Gyr(Torrey et al., 2012).

In observation, the processes of galaxy merging have been probed by statistical studies of galaxy pairs as function of their separation, stellar mass, morphology, mass ratio and many other parameters(e.g. Nikolic et al., 2004; Ellison et al., 2008, 2013). In such studies, a large and unbiased sample of galaxy pairs is crucial. By far, the largest low redshift galaxy pair sample is identified from the main galaxy(MG) sample of the Sloan Digital Sky Survey(SDSS, York et al., 2000), which is a spectroscopic survey of a magnitude-limited sample down to $r<17.77$ (Stoughton et al., 2002). The spectroscopic completeness of the MG sample in SDSS is quite high $(\sim 90 \%$, Hogg et al., 2004). Based on the spectroscopic MGs in the final data release of the SDSS legacy survey(Data Release Seven, DR7 Abazajian et al., 2009), the number of galaxy pairs is over 10,000(e.g. Ellison et al., 2011). Despite of this large number, the galaxy pair sample identified from the spectroscopic MGs is far from complete. The incompleteness of the galaxy pairs is mainly caused by the fiber collision effect in SDSS, which is a minimum separation of 55 arcsec between any two fibers for any given spectroscopic plate. As a result, the completeness of the galaxy pairs identified from the spectroscopic MGs is estimated to be only about 35 percent(Patton \& Atfield, 2008). In other words, the SDSS missed galaxies caused by the fiber collision have a very high probability being in galaxy pairs. Therefore, spectroscopic targeting of the SDSS missed galaxies is an efficient way to identify new galaxy pairs. Only if all these SDSS missed galaxies could be targeted by a new spectroscopic survey, a complete and unbiased galaxy pair sample could be finally made. More importantly, such a sample would be a benchmark on the studies of small-scale environmental effect of low redshift galaxies.

In this manuscript, we introduce the project of observing the SDSS missed MGs with the Guo Shou Jing Telescope (also named as the Large Sky Area Multi-Object Fiber Spectroscopic Telescope - LAMOST) 1 and present its early result: a new sample of galaxy pairs. This paper is organized as follows. In section 2 , we introduce the project of the spectroscopic observation of the SDSS missed MGs with LAMOST. In section 3, we present a new galaxy pair sample using new redshifts from LAMOST survey. Finally, we make short discussions and give summary in Section 4 .

\section{LAMOST SURVEY: COMPLEMENTARY GALAXY SAMPLE}

LAMOST is a special quasi-meridian reflecting Schmidt telescope located at Xinglong Station of National Astronomical Observatory of China. The design of LAMOST provides an effective aperture about 4 meters, a diameter of $\sim 5^{\circ}$ field of view and a spectroscopy system with $\sim 4000$ fibers Wang et al., 1996; Su \& Cui, 2004; Cui, 2012). After about one year of pilot survey (Luo \& et al., 2012), the LAMOST regular spectral survey has started from September of 2012 and would last for 5 five years. An overview of the LAMOST spectral survey can be seen in Zhao et al. (2012). The LAMOST regular survey mainly focuses on Galactic stars, but also includes a significant fraction of extragalactic objects(e.g. Huo, 2013; Shi et al., 2014). One of the sample of the extragalactic sources is the SDSS missed MGs, and is named as the complementary galaxy sample in the LAMOST survey(Luo \& et al., 2015).

\footnotetext{
1 http://www.lamost.org
} 
2.1 the complementary galaxy sample

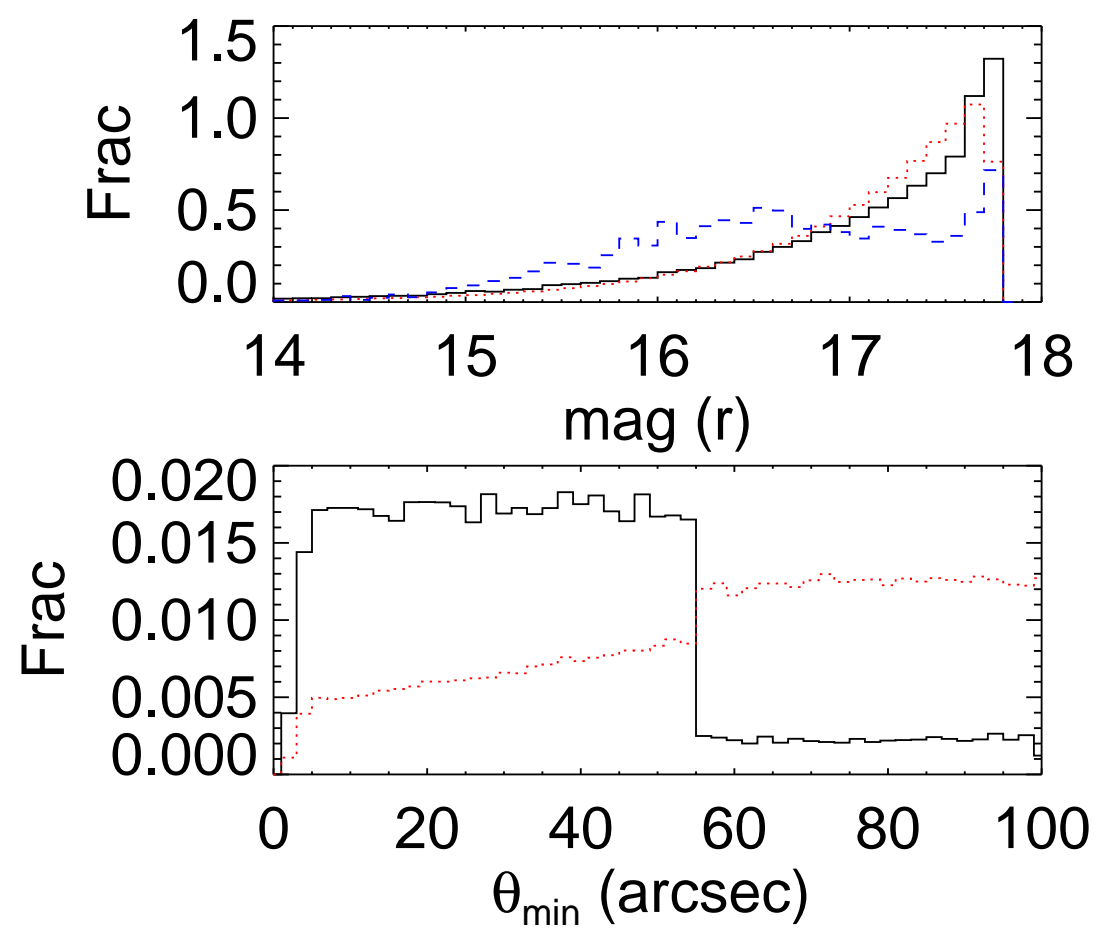

Fig. 1 The SDSS spectroscopic MGs and complementary galaxy sample. Top: the $r$ band Petrosian magnitude distribution. The solid and dotted histograms show the distributions of the complementary galaxies and SDSS spectroscopic MGs respectively, while the dashed histogram shows the 3,456 complementary galaxies with spectra in LAMOST DR2(Section 2.2). Bottom: the angular separation to the nearest SDSS MG(complementary galaxy: solid, SDSS spectroscopic MG: dotted). All the histograms have been normalized to unit area.

The complementary galaxy sample is constructed from the catalog archive server of the SDSS legacy survey, where all the galaxies with $r$ band Petrosian magnitude (Galactic reddening corrected) brighter than $r=17.77$ and not yet with spectroscopic redshifts are selected. The footprint of the complementary galaxies in the LAMOST survey is restricted in the north Galactic cap region $(-10<\delta<60 \mathrm{deg}$ and $b>0 \mathrm{deg})$. 2 After that, we remove a small fraction of galaxy targets that might be contaminated by nearby bright stars using the spherical polygon masks in the NYU value added galaxy catalog (Blanton et al., 2005). The final number of the complementary galaxy sample in the input catalog of the LAMOST spectral survey is 66,263 .

In SDSS DR7, the number of the MGs that have been targeted with spectroscopy in north Galactic cap is 639,428 . That is to say, the fraction of SDSS missed MGs $(66,263$ of 705,691) is about 10 percent. In Fig. 1 we show the $r$ band Petrosian magnitude distributions of the complementary galaxy sample and the SDSS spectroscopic MGs respectively. As can be seen,

\footnotetext{
2 For the south Galactic region, since the SDSS MGs are only located in three stripes, the LAMOST survey includes another independent galaxy spectroscopic survey project, which aims to get the redshifts of of a magnitude-limited sample down to $r<18$.
} 
the complementary galaxies have similar magnitude distribution as the SDSS spectroscopic MGs, but are slightly biased toward faint galaxies. Most of the SDSS missed MGs are due to the fiber collision effect, therefor they are expected to be biased toward the high density region. To show this effect quantitatively, we match each complementary galaxy to the global MG sample and obtain the projected distance $\theta_{\min }$ (in unit of arcsec) to its nearest neighbor. For comparison, we also calculate $\theta_{\min }$ for each SDSS spectroscopic MG. The two distributions of $\theta_{\min }$ are plotted in the bottom panel of Fig. 1. Strong biases of two $\theta_{\min }$ distributions at 55 arcsec are clearly seen. This result not only shows that the complementary galaxies are biased to the high density environment, but also implies that the galaxy pair sample identified from the SDSS spectroscopic sample alone is far from complete(Patton \& Atfield, 2008).

To quantify the incompleteness of the galaxy pairs in SDSS, we select photometric galaxy pairs in the SDSS MG sample, which are defined as the galaxies and their nearest neighbors inside the radii of 100 arsec. We match photometric pairs from the SDSS spectroscopic MGs and from all the MGs respectively. The fraction of the galaxy pairs of these two samples gives us a estimation of the completeness of the galaxy pairs in SDSS spectroscopic MGs. We show the resulted completeness as function of the pair angular separation in the top panel of Fig. 2 Again, we see that a strong incompleteness jumps at the angular separation $\theta<55$ arcsec, where the completeness is only about 30 percent and even decreases with the decreasing of $\theta$. In the bottom panel, we show the completeness of the galaxy pairs as function of the redshift. Here, we have defined the galaxy pairs as these with projected separation $r_{\mathrm{p}}<100 h_{70}^{-1} \mathrm{kpc}$ (see Section 3) and assumed that the separation follows a random distribution. In this case, the galaxy pairs in SDSS spectroscopic MGs decreases with increasing of redshift and reaches a plateau $\sim 30$ percent at $z>0.09$ where the 55 arcsec limit corresponds to a projected distance $100 h_{70}^{-1} \mathrm{kpc}$. Since the peak redshift of the SDSS MGs is at $z \sim 0.1$, the global completeness of the galaxy pairs in SDSS spectroscopic MG sample is less than 40 percent.

\subsection{LAMOST observation}

The complementary galaxies are mixed together with other LAMOST targets(most of them are Galactic stars) and then compiled into the LAMOST survey plates. In each plate, the number density of the complementary galaxies is very low, which are therefore assigned fibers with higher priority than stars. In the LAMOST survey, the input sources are tiled into three different types of plates, the bright(B), medium(M) and faint(F) plates, which are designed to reach the average signal-to-noise ratio $(S / N \sim 10)$ for the objects down to the magnitude limits $r<16.5, r<17.8$ and $r<18.5$ respectively. Most of the complementary galaxies have their magnitudes in the range $16.5<r<17.8$ (Fig. (1) and therefor are mainly tiled into the M plates. On the other hand, due to the limited number of the dark nights, the observing time allocated for the $\mathrm{M}$ plates is quite few. Because of that, only a small fraction of the complementary galaxy sample have been targeted yet.

The spectroscopic data used in this study is from the LAMOST Data Release 2 (DR2), i.e the data till June 2014 . In LAMOST DR2, there are 3,456 complementary galaxies that have been targeted with spectroscopy and with spectra released. The magnitude distribution of these 3,456 LAMOST targeted galaxies is plotted as the dashed histogram in the top panel of Fig. 1. Compared with the input of the 66,263 complementary galaxies, the LAMOST targets are evidently biased toward bright galaxies. There are two reasons for this bias. First, some of the bright complementary galaxies $(r<16.5)$ are compiled into the target list of LAMOST $\mathrm{B}$ plates, which far outnumbers the $\mathrm{M}$ plates. The other reason for the bias against the faint galaxies is the failure of the spectroscopies because of their low $S / N$ (see next section). 

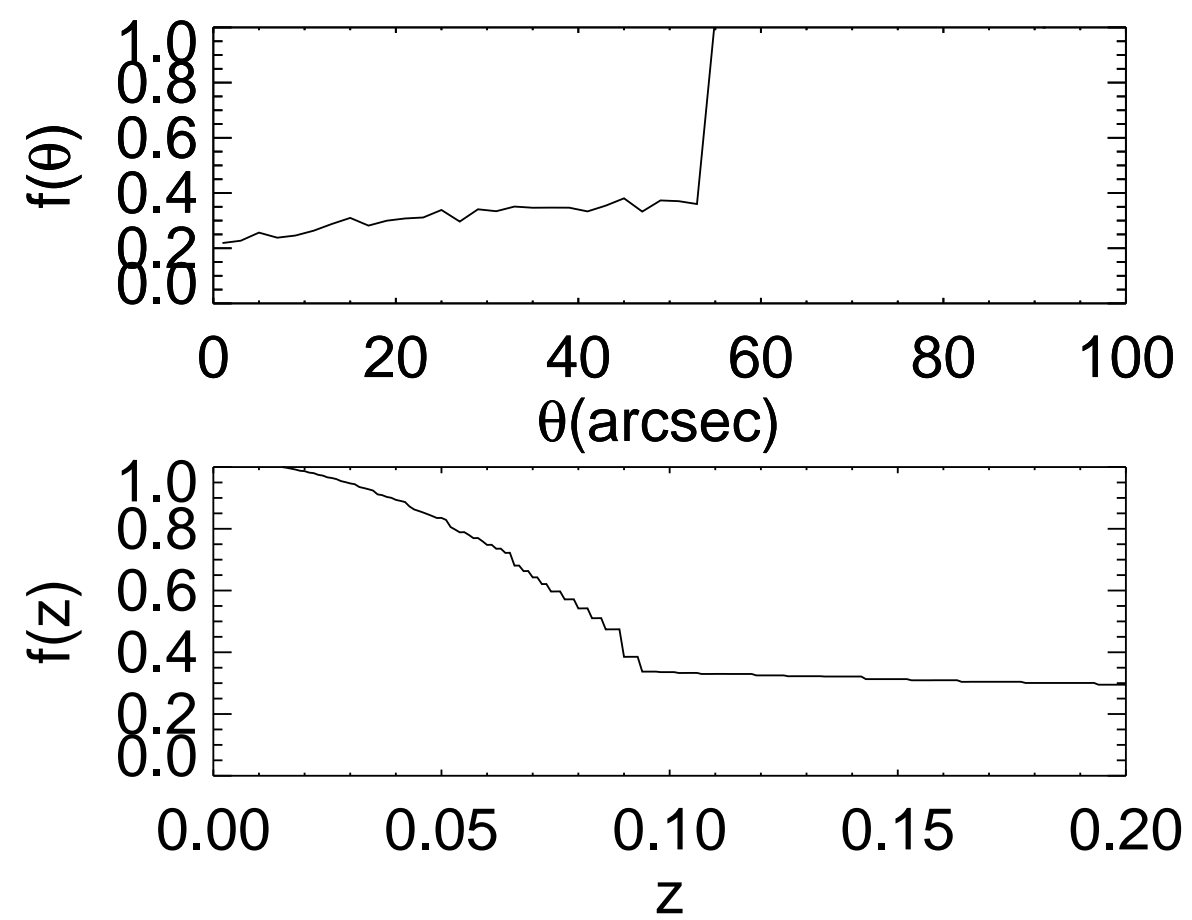

Fig. 2 The completeness of galaxy pairs in SDSS spectroscopic MGs. The top panel shows the completeness as function of the angular separation of the pair members. The bottom panel shows the completeness of the galaxy pairs (defined as these with projected distancer $r_{\mathrm{p}}<100 h_{70}^{-1} \mathrm{kpc}$ ) as function of redshift.

\section{3 redshift measurements}

In the catalog of LAMOST DR2, only 1,951 of the 3456 complementary galaxies have redshifts measured from the LAMOST 1-D pipeline and published in the LAMOST catalog. The failure of the redshift measurements is mainly due to the low $S / N$ of the LAMOST spectra of the faint galaxies $(r>16.5)$. We show the mean $S / N$ in the $r$-band wavelength range against the $r$-band magnitude of the 3,456 LAMOST targeted galaxies in the top panel of Fig. 3. The distribution of the $S / N$ is shown in the bottom panel. As can be seen, when $r>16.5$, the median $S / N$ of the LAMOST spectra becomes lower than 10, which makes the number of the spectra with LAMOST catalog $z$ decreases significantly (see dotted histogram in the bottom panel of Fig. 3).

To further improve the successful rate of the redshift measurement, we developed an independent PCA redshift measurement algorithm following the pipeline used for the 'CMASS' galaxies in the Baryon Oscillation Spectroscopic Survey of the SDSS III(Bolton et al., 2012). The detail of the pipeline will be presented in an upcoming paper. Here, we outline the basic routines in Appendix A. With this algorithm, we obtained 2,796 redshift measurements. In Fig. 3. the dashed line shows the fraction of the spectra with PCA redshifts measured. It is clearly that our new PCA algorithm makes a significant improvement on the redshift measurement of the LAMOST spectra, especially at the low $S / N$ end.

To quantify the redshift measurements from the LAMOST 1-D pipeline and our PCA algorithm, we compare their redshifts with other independent measurements. After the SDSS legacy 


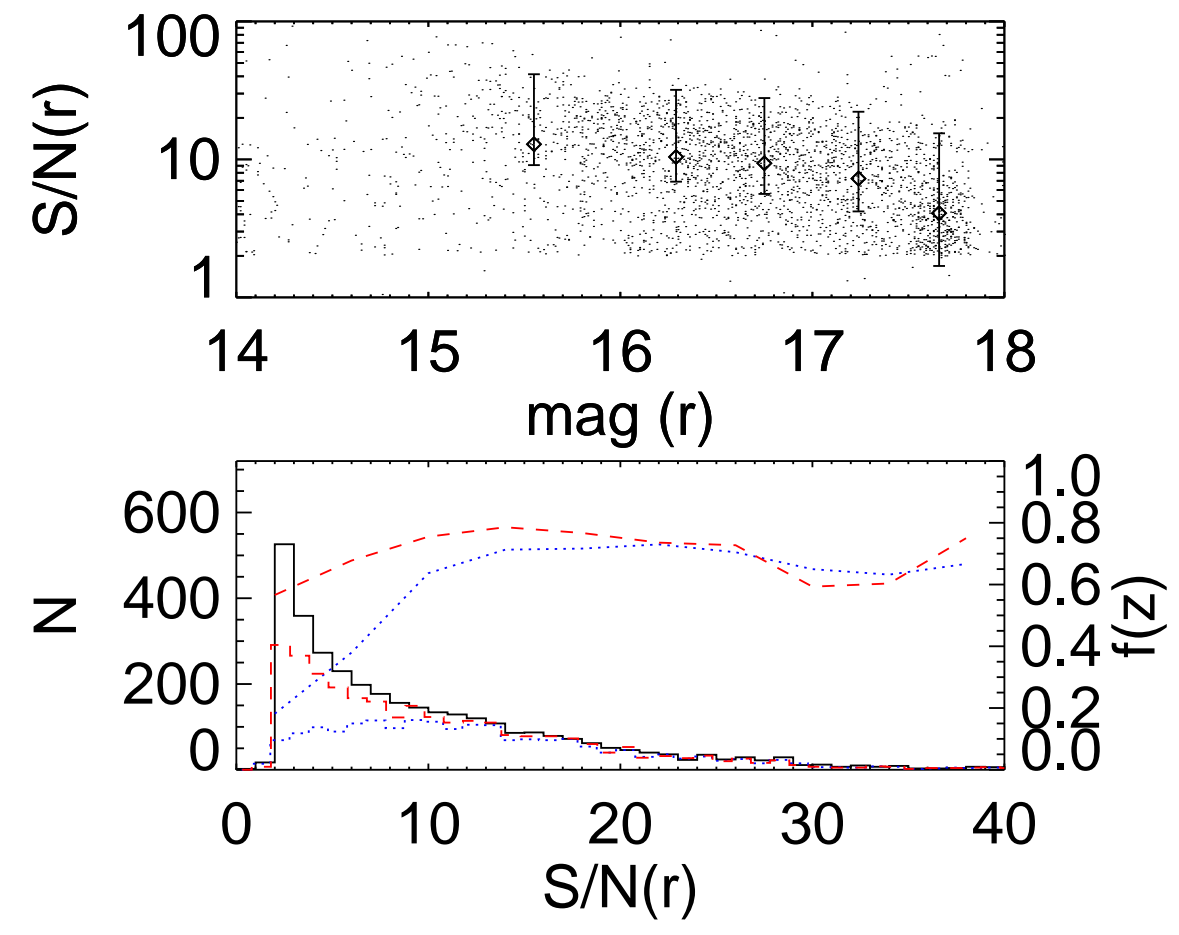

Fig. 3 The signal-to-noise $\operatorname{ratio}(S / N)$ of the LAMOST spectra of the complementary galaxies. The top panel shows the magnitude as function of $S / N$, while the bottom panel shows the histograms of $S / N$. Top: the small dots represent all 3,456 complementary galaxies. The squares with error bars show the median and 16/84 percentiles of the $S / N$ distribution in magnitude bins. Bottom: the solid histogram shows all the 3,456 complementary galaxies, while the dashed and dotted histograms show the sub-samples of galaxies with redshifts measured from the PCA algorithm and form the LAMOST 1-D pipeline respectively. The fractions of galaxies with redshift measured(right $y$-axis) for the spectra at different $S / N$ are shown by the dotted line(PCA algorithm) and dashed line(1-D pipeline).

survey, some of the SDSS missed MGs had been targeted by the BOSS spectroscopy in SDSS III(Dawson et al., 2013) 3. We matched the 3,456 complementary galaxies with SDSS DR12 spectroscopic catalog and obtained 1,056 matches. For these 1,056 galaxies, the LAMOST catalog lists 604 redshifts while our PCA algorithm provides 923 redshift measurements. In Fig. 4. we show the histograms of the differences of the redshifts (in terms of recessional velocity difference $\Delta V$ ) of these galaxies with both LAMOST and SDSS redshifts. The LAOMST redshifts from 1-D pipeline and PCA algorithm both show good consistences with SDSS values. For the LAMOST catalog $z$, the standard deviation of $\Delta V$ is about $80 \mathrm{~km} \mathrm{~s}^{-1}$. For the PCA $z$, the scatter of $\Delta V$ is even smaller, $\sim 58 \mathrm{~km} \mathrm{~s}^{-1}$. Given the better consistence with the SDSS redshifts of the PCA redshift measurements, we use the PCA redshifts for these galaxies with both PCA redshifts and catalog redshifs. For the 1,056 galaxies with SDSS redshifts, we take their redshifts from SDSS catalog. As we will show in the next section, the criterion of the velocity difference we adopted to identify galaxy pairs is $|\Delta V|<500 \mathrm{~km} \mathrm{~s}^{-1}$. Therefore, the

\footnotetext{
3 These galaxies typically have photo flag BRIGHT_GAL in SDSS III.
} 


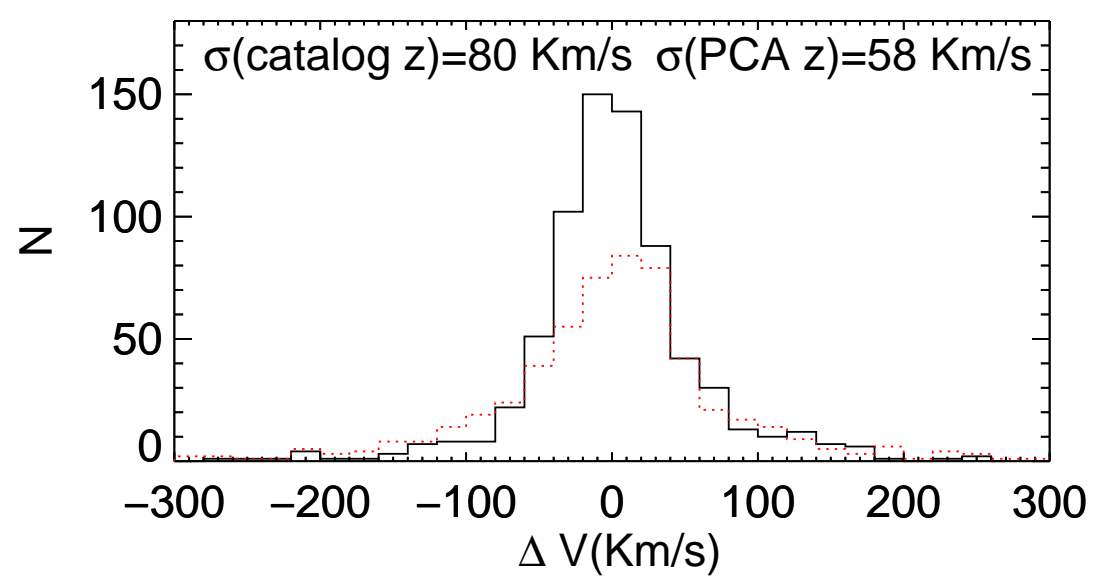

Fig. 4 Number histogram of the redshift differences (in terms of recessional velocity) between the LAMOST and SDSS measurements. The solid histogram shows the differences between the SDSS redshifts and our PCA redshift measurements, while the dotted histogram represents the differences between the SDSS redshifts and and LAMOST 1-D redshifts.

scatter between the SDSS and LAMOST redshift measurements have few impacts on the pair identification.

\section{THE GALAXY PAIR SAMPLE}

In this section, we combine the redshifts of the complementary galaxies with the SDSS spectroscopic MGs to identify new galaxy pairs. For the 3,456 complementary galaxies, we have obtained 3,137 redshifts. Among them, 1,056 redshifts come from SDSS DR12, 1906 from PCA algorithm, 175 from LAMOST 1-D pipeline.

In observation, a galaxy pair is typically defined from the projected distance $r_{\mathrm{p}}$ and recessional velocity difference $|\Delta V|$ of two neighboring galaxies. However, there is no consensus on the critical values of $r_{\mathrm{p}}$ and $|\Delta V|$. For example, both based on SDSS DR7, Liu et al. (2011) defined an AGN pair sample with $|\Delta V|<600 \mathrm{~km} \mathrm{~s}^{-1}$ and $r_{\mathrm{p}}<100 h_{70}^{-1} \mathrm{kpc}$, whereas Patton et al. (2011) searched galaxy pairs using $|\Delta V|<1,000 \mathrm{~km} \mathrm{~s}^{-1}$ and $r_{\mathrm{p}}<80 h_{70}^{-1} \mathrm{kpc}$ (see also Scudder et al., 2012; Argudo-Fernández et al., 2015). For the projected distance $r_{\mathrm{p}}$, there are evidences that galaxies show interactions on their neighbors at $r_{\mathrm{p}}>80 h_{70}^{-1} \mathrm{kpc}$ (Scudder et al., 2012). In this study, we set a critical value $r_{\mathrm{p}}<100 h_{70}^{-1} \mathrm{kpc}$. For $\Delta V$, a large critical value (for example, $|\Delta V|<1,000 \mathrm{~km} \mathrm{~s}^{-1}$ ) might introduce a significant fraction of contamination from the high density environment(e.g. galaxy groups and clusters). In this study, we select galaxy pairs using $|\Delta V|<500 \mathrm{~km} \mathrm{~s}^{-1}$ (see Appendix B for more discussions).

We match the redshifts of 3,137 complementary galaxies with the spectroscopic MGs in SDSS DR7 using the criteria $r_{\mathrm{p}}<100 h_{70}^{-1} \mathrm{kpc}$ and $|\Delta V|<500 \mathrm{~km} \mathrm{~s}^{-1}$ and obtain 1,141 galaxy pair candidates. In a few cases, a complementary galaxy may have more than one SDSS spectroscopic MGs matched. In this case, we choose the galaxy with the smallest $r_{\mathrm{p}}$ as the matched pair member and mark this pair being in a multiple system. We will come to the multiple systems in Section 3.1. Moreover, to have a better quality control to the pair sample, we make visual inspections on the SDSS images for all the pair candidates. In a few cases, the imperfect 

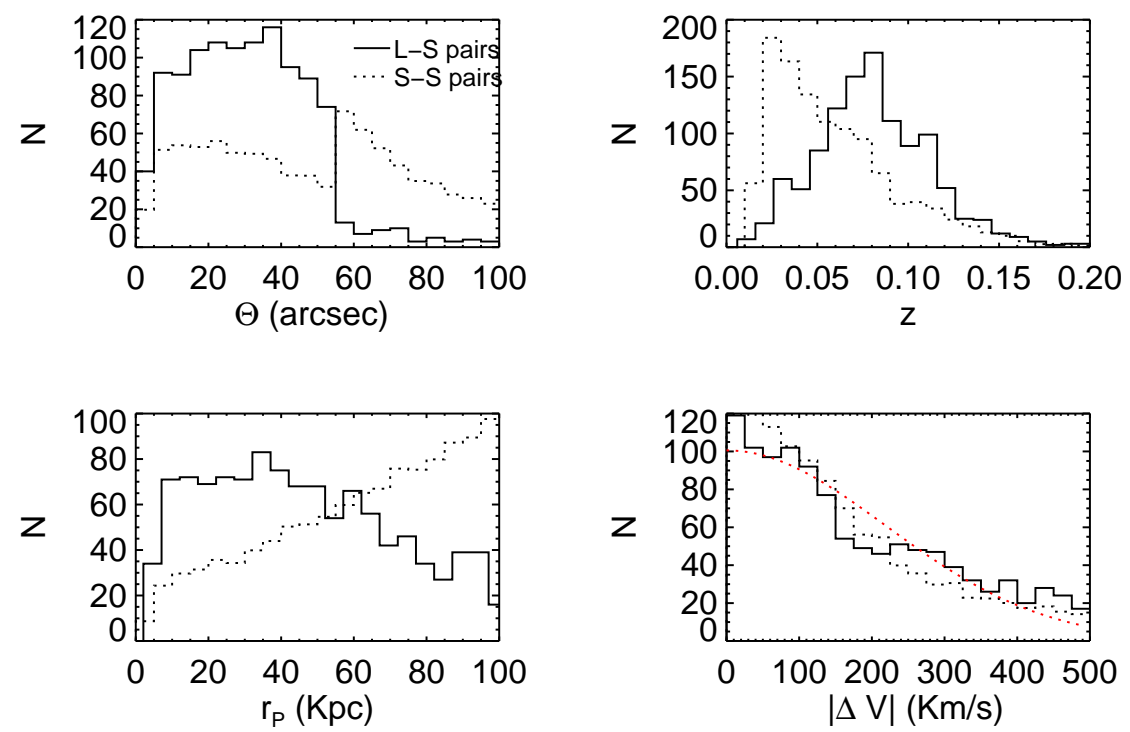

Fig. 5 Basic statistical properties of the 1,102 galaxy pairs identified from the LAMOST complementary galaxies and SDSS MGs(solid histograms). The statistical properties of the SDSS selected galaxy pairs are plotted as the dotted histograms in each panel for comparison (normalized to 1,102). The top left, top right, bottom left and bottom right panels show the histograms of the angular separation, redshift, projected distance and recessional velocity differences respectively. In the bottom right panel, the dotted curve shows a Gaussian distribution function with a standard deviation $218 \mathrm{~km} \mathrm{~s}^{-1}$.

SDSS pipeline de-blends big galaxies into several small children. This effect results in 39 fake pairs. Thus, our final sample include 1,102 galaxy pairs.

We show the histograms of the $r_{\mathrm{p}}$ and $|\Delta V|$ of the final 1,102 galaxy pairs in the bottom two panels of Fig. 5 , where the distributions of the angular separation $\Theta$ (in acrsec) between the pair members and their average redshifts are shown in the top two panels. To have a better understanding of the statistical properties of the new pair sample, we show the distributions of the SDSS only pairs as the dotted histograms in each panel of Fig. 5 for comparison. The SDSS only pairs are selected from the SDSS DR7 group catalog of Yang et al. (2008) using the same criteria above. The number of the SDSS pairs is 16,973. Because of its large number, we have not made visual inspections on this sample. However, according to the fact that there are only 39 of 1,141 LAMOST-SDSS pairs are fake, we expect the impact of the fake pairs to its statistical properties should be quite small. For convenience, we abbreviate the LAMOST-SDSS pairs as the LS pairs and the SDSS only pairs as SS pairs below.

As can be seen from Fig. 5 , except the distribution of $|\Delta V|$, the LS pairs show significant difference from the SS pairs. The angular separation of the SS pairs is clearly biased toward large values $(\Theta>55 \operatorname{arcsec}$, top left panel). This, as we mentioned, is because of the $55 \operatorname{arcsec}$ fiber collision effect in SDSS. The SS pairs are also biased toward lower redshifts(top right panel), which again is because of the fiber collision effect. Although the low $z$ selection effect compensates the high $\Theta$ bias, as a combination, the SS pairs are still biased toward large separation $\left(r_{\mathrm{p}}\right)$ ones (bottom left panel). Compared with the SS pair sample, our new LS pairs only increase the number of pairs by a few percent $(\sim 7 \%)$. However, for the close pairs with 
Table 1 The catalog of galaxy pairs identified from the LAMOST complementary galaxies and SDSS MGs. For each pair, $\alpha_{1}, \delta_{1}, z_{1}$ are the Right Accession, Declination, redshift of the LAMOST complementary galaxy, while $\alpha_{2}, \delta_{2}, z_{2}$ are those of the SDSS galaxy. M_Flag and O_Flag are the multiplicity and overlapping flags (see Section 3.1 and 3.2 for detail). The table is sorted in ascending order of $\alpha_{1}$. The complete table is available on-line.

\begin{tabular}{lllllllll}
\hline ID & $\alpha_{1}[\mathrm{deg}]$ & $\delta_{1}[\mathrm{deg}]$ & $z_{1}$ & $\alpha_{2}[\mathrm{deg}]$ & $\delta_{2}[\mathrm{deg}]$ & $z_{2}$ & M_Flag & O_Flag \\
1 & 112.23653 & 36.91987 & 0.06006 & 112.24269 & 36.91610 & 0.05988 & 0 & 0 \\
2 & 114.54001 & 28.13682 & 0.07852 & 114.54659 & 28.12799 & 0.07942 & 0 & 0 \\
3 & 116.04450 & 23.99016 & 0.07545 & 116.05284 & 23.99939 & 0.07533 & 0 & 0 \\
4 & 116.46624 & 26.47179 & 0.12311 & 116.46654 & 26.47719 & 0.12373 & 0 & 0 \\
5 & 119.86285 & 23.97182 & 0.09218 & 119.85204 & 23.98273 & 0.09318 & 0 & 0 \\
6 & 120.09847 & 39.83033 & 0.01320 & 120.17009 & 39.87050 & 0.01326 & 1 & 0 \\
7 & 120.40500 & 15.70968 & 0.01545 & 120.36157 & 15.74989 & 0.01637 & 1 & 0 \\
8 & 120.83544 & 23.96435 & 0.05784 & 120.84409 & 23.96933 & 0.05725 & 0 & 0 \\
9 & 120.89831 & 28.54465 & 0.14178 & 120.89262 & 28.55000 & 0.14096 & 0 & 0 \\
10 & 121.02204 & 31.44029 & 0.07302 & 121.03594 & 31.43697 & 0.07303 & 0 & 0 \\
11 & 121.37741 & 22.13299 & 0.13852 & 121.38057 & 22.12452 & 0.14003 & 0 & 0 \\
12 & 123.44543 & 8.38181 & 0.11409 & 123.44723 & 8.38925 & 0.11324 & 0 & 0 \\
13 & 123.98402 & 8.28538 & 0.14295 & 123.97318 & 8.28571 & 0.14438 & 0 & 0 \\
14 & 124.05299 & 3.85966 & 0.08668 & 124.05083 & 3.85807 & 0.08777 & 0 & 1 \\
15 & 124.46973 & 7.57755 & 0.12465 & 124.46754 & 7.57477 & 0.12484 & 0 & 0 \\
\hline
\end{tabular}

more significant interaction $\left(r_{\mathrm{p}}<30 h_{70}^{-1} \mathrm{kpc}\right)$, the number of the LS pairs enlarges the SS pairs by more than 14 percent(358 versus 2527). Therefor, the LS pair sample could be a useful supplement to the current SS pair sample, especially for the close pairs, which are valuable in the statistical studies of galaxy interaction and merging .

For the velocity difference (bottom right panel), the standard deviation of the LS and SS pairs is 218 and $198 \mathrm{~km} \mathrm{~s}^{-1}$ respectively. Considering the fact that the dispersion of the redshift differences between the LAMOST and SDSS measurements could be as large as $80 \mathrm{~km} \mathrm{~s}^{-1}$ (Fig. 4), these two results are consistent with each other. In this panel, we also plot a Gaussian distribution with standard deviation $\sigma=218 \mathrm{~km} \mathrm{~s}^{-1}$ for comparison. As we can see, the distribution of $|\Delta V|$ of LS pairs deviates from the Gaussian function, especially at the tails (see Appendix B for a more detailed discussion).

The catalog of the LS pairs are listed in Table 1. Besides basic parameters(e.g. Ra, Dec, redshift) for each pair member, we also list two extra flags(M_Flag and O_Flag) for each pair, which characterizes the multiplicity and the image overlapping of the pair members respectively. In the following two sub-sections, we make brief descriptions on these two flags.

\section{1 multiple system}

Our galaxy pair sample is defined from two simple observational criteria $\left(r_{\mathrm{p}}<100 h_{70}^{-1} \mathrm{kpc}\right.$ and $\left.|\Delta V|<500 \mathrm{~km} \mathrm{~s}^{-1}\right)$. Besides the pair members, the other neighboring galaxies have not been taken into consideration. However, to study the galaxy interaction and galaxy merging using galaxy pairs, it is better to refine a sample of physical pairs, where the interaction between two pair members overtakes the effects from other neighbors(e.g. Argudo-Fernández et al., 2015). For this purpose, we mark the galaxy pairs in multiple system with a multiple flag 'M_Flag'. Our aim is to remind that these pairs may not be suitable for studying galaxy interactions by only considering their members.

We define a galaxy pair in multiple system when either of its member has another main galaxy neighbor $(r<17.77)$ that also satisfies the pair definition $\left(r_{\mathrm{p}}<100 h_{70}^{-1} \mathrm{kpc}\right.$ and $|\Delta V|<$ $500 \mathrm{~km} \mathrm{~s}^{-1}$ ). Within current data, 197 of the 1,102 LS pairs are found in multiple systems. 


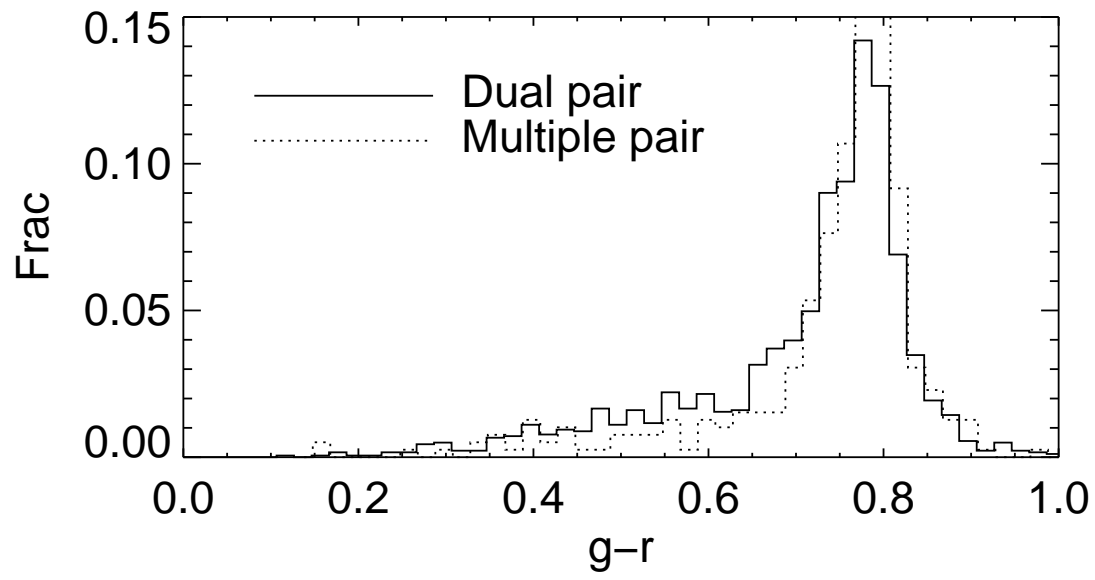

Fig. 6 Distribution of the the $g-r$ color of the pair members. The solid and dotted histograms (normalized to unit area) show the galaxies in the pairs with M_Flag $=0$ (dual) and M_Flag = 1 (multiple) respectively.

These pairs are marked with flag M_Flag $=1$ in Table 1 and noted as 'multiple' pairs below. For comparison, the pairs with M_Flag $=0$ are noted as 'dual' pairs.

To check the possible distinction between the multiple and dual pairs, we compare the $g-r$ color(taking from the SDSS model magnitudes and with $K$-correction applied) distributions of their members in Fig. 6. The galaxies in multiple pairs are averagely redder than in dual ones. In specific, the red galaxy fraction $(g-r>0.7)$ in the dual and multiple pairs are 0.52 and 0.66 respectively. This color bias is caused by the fact that the multiple pairs are biased toward high density environment (i.e. galaxy groups and clusters).

We remind that the M_Flag set for the multiple systems is quite a preliminary parameter. Since the pairs are selected from a magnitude-limited sample, both of the pair sample and the multiplicity flag have strong redshift dependence. For example, a triplet system with $M_{\mathrm{r}, i}=-20,-21,-22 \mathrm{mag}$ would be identified as a triplet, a pair and a single galaxy in the SDSS MG sample $(r<17.77)$ at redshift $z=0.05,0.1,0.15$ respectively. On the other hand, the multiplicity flag is also not complete, which is because of the high incompleteness of the SDSS spectroscopic MGs at small scales and also because of the small fraction of the complementary galaxies been targeted by LAMOST yet. For the dual pairs with M_Flag $=0$, they are still possibly in multiple systems, i.e. having another companion galaxy but without redshift measured yet 4 That is to say, the fraction of the pairs in multiple system (197 of 1102) is actually a lower limit. Given the significant fraction of galaxy pairs in multiple system, the studies of galaxy interactions with galaxy pairs should take the multiplicity of the galaxy systems into consideration(Shen et al. 2015, in preparation).

\section{2 overlapping pairs}

The luminosity/mass ratio of the pair members plays an important role in galaxy merging(Jiang et al., 2014). However, photometry of a very close galaxy pair is a nontrivial

\footnotetext{
4 The pair with $\mathrm{ID}=52$ in Table 1 is actually an isolated and compact galaxy triplet, which is found to be a triplet candidate during our visual inspection and then spectroscopically confirmed by a follow-up observation with the $2.16 \mathrm{~m}$ telescope in the Xinglong Station(Feng et al. , 2015) .
} 


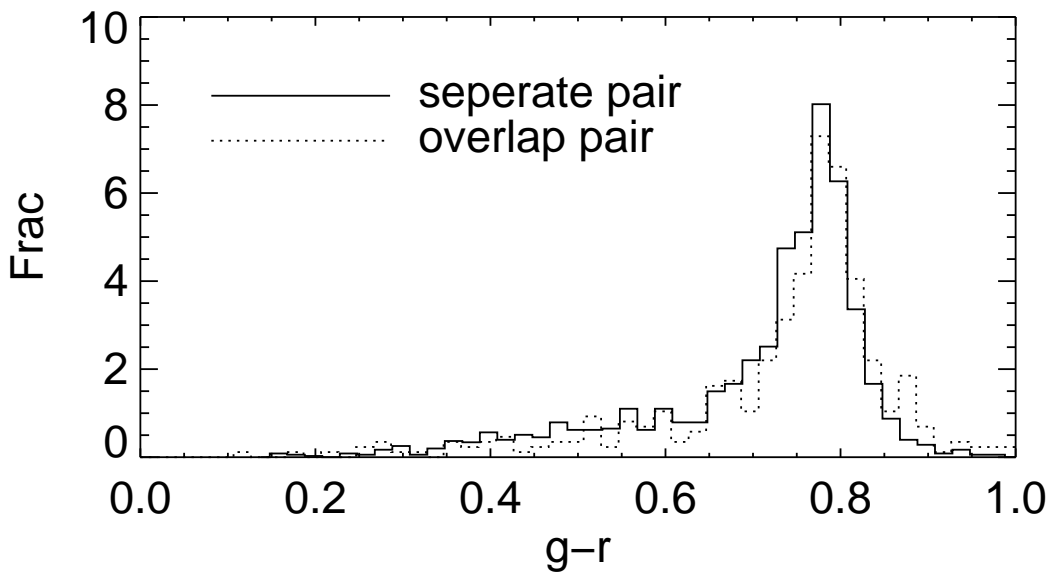

Fig. 7 Distribution of the $g-r$ color of the pair members. The solid and dotted histograms(normalized to unit area) show the galaxies in the pairs with O_Flag $=0$ and O_Flag $=1$ respectively.

task(Simard et al., 2011). During the visual inspection of the galaxy pairs, we noticed some of the galaxy pairs whose images of their members overlap each other significantly. We mark such pairs $(N=216)$ with a flag O_Flag $=1$. For these overlapping pairs, the uncertainties of their photometry might introduce uncertainties and possible biases in the estimation of the stellar mass and mass ratio.

We show the $g-r$ color distribution of the overlapping pairs as the dotted histogram in Fig. 7. where the distribution of the other pairs is shown as the solid histogram for comparison. As can be seen, although the overlapping pairs have much closer projected distance, their colors are biased toward redder values. The fraction of red galaxies $(g-r>0.70)$ in the overlapping pairs is 62 percent, which is significantly higher than the fraction 0.52 for the non-overlapping pairs. Unless there are big uncertainties in the photometry of these overlapping pairs, it is hard to explain the color bias we see in Fig. 7 Actually, Patton et al. (2011) have already shown that the poor photometry of SDSS official pipeline is largely responsible for the suspicious and large fraction of of extreme red galaxies(e.g. $g-r>0.9$ ) in the very close galaxy pairs. Therefore, to get a better estimation of the mass ratio of these overlapping pairs, more detailed photometry is required(e.g. Simard et al., 2011). Here, we set this flag for a caution and leave the detailed photometry for a future work.

\section{CONCLUSION}

In this paper, we presented the project of the spectroscopic survey of the SDSS missed MGs $(r<$ 17.77) using LAMOST. The SDSS missed MGs are named as the complementary galaxy sample in the LAMOST survey. In the first two years of the LAMOST survey, due to the limited survey time of the medium(M) plates, only a small fraction $(3,456$ of 66,263$)$ of the complementary galaxies had obtained LAMOST spectra. The majority of these spectra have quite low $S / N$, which are mostly due to the poor seeing condition. We developed a PCA algorithm to improve the redshift measurements of these low $S / N$ spectra. Together with the SDSS DR12 match and LAMOST 1-D pipeline results, we finally obtained 3,137 redshifts of the 3,456 complementary galaxies. 
Considering the fact that the SDSS missed galaxies are mainly caused by the fiber collision effect, the spectroscopy of the complementary galaxies has a great potential in identifying new galaxy pairs. We present such a catalog of galaxy pairs identified from the first two years data of the LAMOST survey. From the redshifts of 3,137 complementary galaxies, we obtained a sample of 1,102 galaxy pairs after a careful visual inspection. Compared with the galaxy pairs selected from SDSS data only, our pair sample includes a larger fraction of close pairs $\left(r_{\mathrm{p}}<30 h_{70}^{-1} \mathrm{kpc}\right)$. Because of such advantages, our sample increased the current SDSS close pairs sample by about an amount of $\sim 15$ percent. In common with other studies, our pairs are selected using two simple observational criteria $\left(r_{\mathrm{p}}\right.$ and $\left.|\Delta V|\right)$. Whether they are physical bounding systems have not been taken into consideration. We find that at least $\sim 20$ percent of the pairs are actually located in multiple systems $(($ M_Flag $=1))$. Moreover, during the visual inspection, we find, for about 20 percent the pairs, the images of their members overlap each other. Therefor, the photometry and stellar mass estimation of the galaxies in these overlapping pairs(O_Flag =1) should be in caution.

With the acceleration of the LAMOST survey on the complementary galaxies in the current and future season(from Sep. of 2014), we expect that the completeness of the galaxy pairs will be further improved. Once the LAMOST survey had finished the spectroscopies of the complementary galaxy sample, the great progresses on the studies of galaxy pair, galaxy interactions, galaxy merging and the small-scale environmental effects of galaxies would be expected.

Acknowledgements Guoshoujing Telescope (the Large Sky Area Multi-Object Fiber Spectroscopic Telescope, LAMOST) is a National Major Scientific Project built by the Chinese Academy of Sciences. Funding for the project has been provided by the National Development and Reform Commission. LAMOST is operated and managed by the National Astronomical Observatories, Chinese Academy of Sciences.

This work is supported by the "973 Program" 2014 CB845705, Strategic Priority Research Program "The Emergence of Cosmological Structures" of the Chinese Academy of Sciences (CAS; grant XDB09030200) and the National Natural Science Foundation of China (NSFC) with the Project Number of 11573050 and 11433003.

\section{References}

Abazajian, K. N., Adelman-McCarthy, J. K., Agüeros, M. A., Allam, S. S., Allende Prieto, C., An, D., Anderson, K. S. J., Anderson, S. F., Annis, J., Bahcall, N. A., \& et al. 2009, ApJS, 182,543

Argudo-Fernández, M., Verley, S., Bergond, G., Duarte Puertas, S., Ramos Carmona, E., Sabater, J., Fernández Lorenzo, M., Espada, D., Sulentic, J., Ruiz, J. E., \& Leon, S. 2015, ArXiv e-prints

Blanton, M. R., Schlegel, D. J., Strauss, M. A., Brinkmann, J., Finkbeiner, D., Fukugita, M., Gunn, J. E., Hogg, D. W., Ivezić, Ž., Knapp, G. R., Lupton, R. H., Munn, J. A., Schneider, D. P., Tegmark, M., \& Zehavi, I. 2005, AJ, 129, 2562

Bolton, A. S., Schlegel, D. J., Aubourg, E., Bailey, S., Bhardwaj, V., Brownstein, J. R., Burles, S., Chen, Y.-M., Dawson, K., Eisenstein, D. J., Gunn, J. E., Knapp, G. R., Loomis, C. P., Lupton, R. H., Maraston, C., Muna, D., Myers, A. D., Olmstead, M. D., Padmanabhan, N., Pâris, I., Percival, W. J., Petitjean, P., Rockosi, C. M., Ross, N. P., Schneider, D. P., Shu, Y., Strauss, M. A., Thomas, D., Tremonti, C. A., Wake, D. A., Weaver, B. A., \& Wood-Vasey, W. M. 2012, AJ, 144, 144

Cui, X.-Q. e. a. 2012, Research in Astronomy and Astrophysics, 12, 1197

Dawson, K. S., Schlegel, D. J., Ahn, C. P., Anderson, S. F., Aubourg, É., Bailey, S., Barkhouser, R. H., Bautista, J. E., Beifiori, A., Berlind, A. A., Bhardwaj, V., Bizyaev, D., Blake, C. H., Blanton, M. R., Blomqvist, M., Bolton, A. S., Borde, A., Bovy, J., Brandt, W. N., Brewington, H., Brinkmann, J., Brown, P. J., Brownstein, J. R., Bundy, K., Busca, N. G., Carithers, W., 
Carnero, A. R., Carr, M. A., Chen, Y., Comparat, J., Connolly, N., Cope, F., Croft, R. A. C., Cuesta, A. J., da Costa, L. N., Davenport, J. R. A., Delubac, T., de Putter, R., Dhital, S., Ealet, A., Ebelke, G. L., Eisenstein, D. J., Escoffier, S., Fan, X., Filiz Ak, N., Finley, H., FontRibera, A., Génova-Santos, R., Gunn, J. E., Guo, H., Haggard, D., Hall, P. B., Hamilton, J.-C., Harris, B., Harris, D. W., Ho, S., Hogg, D. W., Holder, D., Honscheid, K., Huehnerhoff, J., Jordan, B., Jordan, W. P., Kauffmann, G., Kazin, E. A., Kirkby, D., Klaene, M. A., Kneib, J.-P., Le Goff, J.-M., Lee, K.-G., Long, D. C., Loomis, C. P., Lundgren, B., Lupton, R. H., Maia, M. A. G., Makler, M., Malanushenko, E., Malanushenko, V., Mandelbaum, R., Manera, M., Maraston, C., Margala, D., Masters, K. L., McBride, C. K., McDonald, P., McGreer, I. D., McMahon, R. G., Mena, O., Miralda-Escudé, J., Montero-Dorta, A. D., Montesano, F., Muna, D., Myers, A. D., Naugle, T., Nichol, R. C., Noterdaeme, P., Nuza, S. E., Olmstead, M. D., Oravetz, A., Oravetz, D. J., Owen, R., Padmanabhan, N., Palanque-Delabrouille, N., Pan, K., Parejko, J. K., Pâris, I., Percival, W. J., Pérez-Fournon, I., Pérez-Ràfols, I., Petitjean, P., Pfaffenberger, R., Pforr, J., Pieri, M. M., Prada, F., Price-Whelan, A. M., Raddick, M. J., Rebolo, R., Rich, J., Richards, G. T., Rockosi, C. M., Roe, N. A., Ross, A. J., Ross, N. P., Rossi, G., Rubiño-Martin, J. A., Samushia, L., Sánchez, A. G., Sayres, C., Schmidt, S. J., Schneider, D. P., Scóccola, C. G., Seo, H.-J., Shelden, A., Sheldon, E., Shen, Y., Shu, Y., Slosar, A., Smee, S. A., Snedden, S. A., Stauffer, F., Steele, O., Strauss, M. A., Streblyanska, A., Suzuki, N., Swanson, M. E. C., Tal, T., Tanaka, M., Thomas, D., Tinker, J. L., Tojeiro, R., Tremonti, C. A., Vargas Magaña, M., Verde, L., Viel, M., Wake, D. A., Watson, M., Weaver, B. A., Weinberg, D. H., Weiner, B. J., West, A. A., White, M., Wood-Vasey, W. M., Yeche, C., Zehavi, I., Zhao, G.-B., \& Zheng, Z. 2013, AJ, 145, 10

Diaferio, A. \& Geller, M. J. 1996, ApJ, 467, 19

Ellison, S. L., Mendel, J. T., Patton, D. R., \& Scudder, J. M. 2013, MNRAS, 435, 3627

Ellison, S. L., Patton, D. R., Mendel, J. T., \& Scudder, J. M. 2011, MNRAS, 418, 2043

Ellison, S. L., Patton, D. R., Simard, L., \& McConnachie, A. W. 2008, AJ, 135, 1877

Hogg, D. W., Blanton, M. R., Brinchmann, J., Eisenstein, D. J., Schlegel, D. J., Gunn, J. E., McKay, T. A., Rix, H.-W., Bahcall, N. A., Brinkmann, J., \& Meiksin, A. 2004, ApJ, 601, L29

Huo, Z.-Y. e. a. 2013, AJ, 145, 159

Jiang, C. Y., Jing, Y. P., \& Han, J. 2014, ApJ, 790, 7

Liu, X., Shen, Y., Strauss, M. A., \& Hao, L. 2011, ApJ, 737, 101

Luo, A.-L. \& et al. 2012, Research in Astronomy and Astrophysics, 12, 1243

Luo, A.-L. \& et al. 2015, Research in Astronomy and Astrophysics, 15, 1095

Nikolic, B., Cullen, H., \& Alexander, P. 2004, MNRAS, 355, 874

Patton, D. R. \& Atfield, J. E. 2008, ApJ, 685, 235

Patton, D. R., Ellison, S. L., Simard, L., McConnachie, A. W., \& Mendel, J. T. 2011, MNRAS, 412,591

Scudder, J. M., Ellison, S. L., Torrey, P., Patton, D. R., \& Mendel, J. T. 2012, MNRAS, 426, 549

Sheth, R. K. 1996, MNRAS, 279, 1310

Shi, Z.-X., Luo, A.-L., Comte, G., Chen, X.-Y., Wei, P., Zhao, Y.-H., Wu, F.-C., Zhang, Y.-X., Shen, S.-Y., Yang, M., Wu, H., Wu, X.-B., Zhang, H.-T., Lei, Y.-J., Zhang, J.-N., Wang, T.-G., Jin, G., \& Zhang, Y. 2014, Research in Astronomy and Astrophysics, 14, 1234

Simard, L., Mendel, J. T., Patton, D. R., Ellison, S. L., \& McConnachie, A. W. 2011, ApJS, 196,11

Springel, V., Di Matteo, T., \& Hernquist, L. 2005, MNRAS, 361, 776

Stoughton, C., Lupton, R. H., Bernardi, M., Blanton, M. R., Burles, S., Castander, F. J., Connolly, A. J., Eisenstein, D. J., Frieman, J. A., Hennessy, G. S., Hindsley, R. B., Ivezić, Ž., Kent, S., Kunszt, P. Z., Lee, B. C., Meiksin, A., Munn, J. A., Newberg, H. J., Nichol, R. C., Nicinski, T., Pier, J. R., Richards, G. T., Richmond, M. W., Schlegel, D. J., Smith, J. A., \& Strauss, M. A. 2002, AJ, 123, 485 
Su, D.-Q. \& Cui, X.-Q. 2004, ChJAA (Chin. J. Astron. Astrophys.), 4, 1

Torrey, P., Cox, T. J., Kewley, L., \& Hernquist, L. 2012, ApJ, 746, 108

Wang, S.-G., Su, D.-Q., Chu, Y.-Q., Cui, X., \& Wang, Y.-N. 1996, Appl. Opt., 35, 5155

Yang, X., Mo, H. J., \& van den Bosch, F. C. 2008, ApJ, 676, 248

York, D. G., Adelman, J., Anderson, Jr., J. E., Anderson, S. F., Annis, J., Bahcall, N. A., Bakken, J. A., Barkhouser, R., Bastian, S., Berman, E., Boroski, W. N., Bracker, S., Briegel, C., Briggs, J. W., Brinkmann, J., Brunner, R., Burles, S., Carey, L., Carr, M. A., Castander, F. J., Chen, B., Colestock, P. L., Connolly, A. J., Crocker, J. H., \& Csabai, I. 2000, Astro. J., 120,1579

Zhao, G., Zhao, Y.-H., Chu, Y.-Q., Jing, Y.-P., \& Deng, L.-C. 2012, Research in Astronomy and Astrophysics, 12, 723

\section{Appendix A: REDSHIFT MEASUREMENTS WITH PCA EIGEN-TEMPLATES}

We developed an independent algorithm to measure the redshifts of the LAMOST complementary galaxies using PCA eigen-templates and chi-squared fitting.

The eigen-templates are derived from the 1,056 complementary galaxies with DR12 redshifts. In specific, we first divide 1,056 galaxies into $20 \mathrm{~g}-r$ color bins with similar numbers. Then, using the SDSS DR12 redshifts, we stack their LAMOST spectra and build the LAMOST composite spectra for galaxies in each $g-r$ bin. We make a PCA decomposition for these 20 composite spectra and find that the first 4 eigenvectors can recover most of the spectroscopic features.

We explore the redshift of each galaxy in the range $0.005<z<0.5$ by taking trial values that are moved with steps of each spectrum pixels. For each trial redshift, we fit the observed spectrum with the error-weighted least-square linear combination of the four 'eigen-spectra' and a four-order poly-nominal. The poly-nominal is introduced to compensate the calibration of the LAMOST spectrum. The reduced $\chi^{2}$ value(the resulted $\chi^{2}$ divided by the number of fitting pixels) for each trial redshift define a $\chi^{2}(z)$ curve in the probed redshift range. The best redshift estimation is then defined by the minimal of the $\chi^{2}(z)$ curve. The error is evaluated at the location where the $\chi^{2}$ is increased by one at each side of the minimum values. During the fitting, besides the pixels with ANDMASK set, we also have masked the pixels at the wavelengths where the sky-subtraction residuals are 3 times more than the average noise.

\section{Appendix B: THE PAIR-WISE PECULIAR VELOCITY DISTRIBUTION OF GALAXY PAIRS}

The distribution of the recessional velocity difference between the galaxy pair members, also known as the pair-wise peculiar velocity distribution function(PVDF), plays a very important role in the large scale structure studies. On the non-linear scales $(r<1 \mathrm{Mpc})$, both observations and theoretical models suggest an exponential PVDF, which can be explained and approximated by a weighted integral of Gaussian distributions of subunits(e.g. Diaferio \& Geller, 1996; Sheth, 1996). However, the PVDF of the pairs in our analysis is on very small scale, even smaller than the viral radius of galaxies $(r<100 \mathrm{kpc})$, which has not drawn much attention in related studies.

We show the pair-wise peculiar velocity $(\Delta V)$ distribution of the SDSS selected pairs in Fig. B.1. The SDSS pairs are selected with criteria $r_{\mathrm{p}}<100 \mathrm{kpc}$ and $|\Delta V|<800 \mathrm{~km} \mathrm{~s}^{-1}$. As we can see, the $\Delta V$ distribution shows long tails out to $\pm 800 \mathrm{~km} \mathrm{~s}^{-1}$ and can not be fitted well by a Gaussian function. We first fit the observed $\Delta V$ distribution with the usually adopted exponential profile,

$$
f(\Delta V)=\frac{1}{\sqrt{2} \sigma} \exp \left(-\frac{\sqrt{2} \Delta V}{\sigma}\right),
$$


where $\sigma$ characterizes the pair-wise peculiar velocity dispersion. The best fitting of the exponential profile has $\sigma=324 \mathrm{~km} \mathrm{~s}^{-1}$ and is shown as the blue line in Fig. B.1. Although better than the Gaussian profile fitting, the exponential profile still can not fit well the fine structure of the observed $\Delta V$ distribution, especially at $|\Delta V|>200 \mathrm{~km} \mathrm{~s}^{-1}$ ranges.

Alternatively, as a preliminary test, we fit the observed $\Delta V$ distribution with a multicomponent model. The observed $\Delta V$ distribution might be contributed by different components and each component corresponds to different physical circumstances. The ideal case of a galaxy pair is that the pair members form a gravitational bound system. In this case, $\Delta V$ represents the orbital velocity of the pair members. A more common case of the observed galaxy pair is that the galaxy pairs have other close neighbors. That is to say, the pair members and their neighbors together locate in a bigger galaxy system, e.g. galaxy groups and clusters. In this case, the pair members orbit individually in the gravitational potential of the host halo and $\Delta V$ represents its velocity dispersion. Finally, $\Delta V$ may be dominated by the difference of the Hubble flow. In this case, the radial distance of the members $(\sim \mathrm{Mpc})$ is much larger than the tangential distance $(<100 \mathrm{kpc})$. That is to say, the 'pair' members actually have few physical interactions on each other and such pairs could be considered as contaminations from projection. Motivated by above scenario, we fit the observed $\Delta V$ distribution with three components, one narrow Gaussian profile(orbital velocities of ideal pairs), one broad Gaussian profile(velocity dispersion of host halos) and a flat component(projection contamination). The best fitting of this three-component model is shown as the solid line in Fig. B.1 As we can see, this model makes a pretty good fitting to the observed $\Delta V$ distribution. The $\Delta V$ profiles of the three components are shown as the dotted lines in Fig. B.1. The narrow Gaussian component has a standard deviation $\sim 110 \mathrm{~km} \mathrm{~s}^{-1}$ and contributes about 40 percent of the galaxy pairs with $|\Delta V|<800 \mathrm{~km} \mathrm{~s}^{-1}$. The broad component has a deviation $\sim 300 \mathrm{~km} \mathrm{~s}^{-1}$ and makes a half contribution. The constant component contributes the last 10 percent.

In Section 3.2, we select the LAMOST-SDSS galaxy pairs with criteria $r_{\mathrm{p}}<100 \mathrm{kpc}$ and $|\Delta V|<500 \mathrm{~km} \mathrm{~s}^{-1}$. Our motivation of choosing a smaller critical value of $|\Delta V|$ is to reduce the contribution from galaxy groups and clusters, i.e. the broad component in Fig. B.1, while include all possible circumstances of ideal pairs. A more detailed study on the $\Delta V$ distribution of the galaxy pairs in different environments is in preparation. 


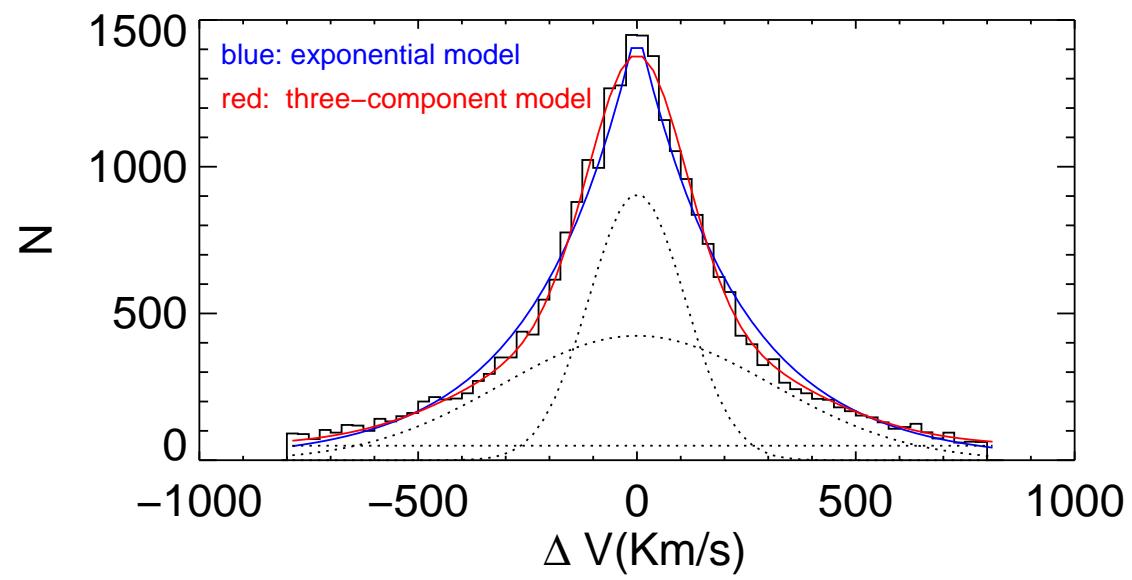

Fig. B.1 Recessional velocity difference $\Delta V$ distribution of the galaxy pairs in SDSS. The best exponential model fitting is shown as the blue line. The best three-component model fitting is shown as the red line, where the contributions of each component are shown as the dotted lines. 